\title{
STUDIES IN TEMPERATURE SENSATION. II. THE TEMPERATURE CHANGES RESPONSIBLE FOR THE STIMULATION OF THE HEAT END ORGANS
}

\author{
By THEODORE W. OPPEL 1 AND JAMES D. HARDY \\ (From the Russell Sage Institute of Pathology in affiliation with the New York Hospital, and \\ the Department of Medicine, Cornell University Medical College, New York City)
}

(Received for publication January 6, 1937)

In the first paper of this series (10) it was shown that the sensation produced by radiation depends on the absorption of the radiant energy by the skin and is the result of stimulation of the heat end organs. Although many workers have studied temperature sensation there is still no uniform opinion as to what thermal changes activate these end organs. The sensation has been thought to depend on the degree of temperature change (7), the rate of temperature change (12), or on alteration of the normal temperature relationships in the different layers of the skin (4). Bazett (1) and his associates have made the most extensive recent study. They identified the Krause end organs which lie $0.1 \mathrm{~mm}$. deep in the skin, as the warm receptors, and the Ruffini organs at $0.3 \mathrm{~mm}$. as the cold receptors (2), and with small thermocouples placed in the skin attempted to correlate the temperature changes at the end organs with sensation (3). The data did not support any of the common theories of thermal end organ response and could not be explained satisfactorily by any other hypothesis.

An adaptation of the technique that we used in the study of the radiation of heat from the human body provides a new approach to the study of temperature sensation. Without touching the skin, we heated it and measured its surface temperature. The measurements were made by a radiometer designed to determine the skin temperature by measuring the radiation from its surface. This instrument has been found to be more accurate than any others used to measure skin temperature (6). By using radiation to heat the skin, the number of calories applied per second could be readily measured. Furthermore, different types of radiation produce different combinations of deep and surface heating. The penetrating radiation is partly absorbed below the surface so that the deep tem-

1 New York Hospital Research Fellow. perature is elevated at the same time as the surface temperature. Non-penetrating radiation heats only the skin surface. The thermal gradients in the skin could thus be altered by the use of the various types of radiation. While such gradient changes cannot be measured, they can be roughly estimated on the basis of the penetrating properties of the several types of radiant energy. The penetrating properties of visible radiation, penetrating infra-red radiation, and non-penetrating infra-red radiation for human skin were reviewed in the first paper of this series and shown to be quite different. The skin temperature elevations which occurred when minimal sensation is produced by these types of radiation were compared in the present study.

\section{METHODS}

The sources of radiation and the methods of measuring the minimum stimulus and the reflecting power of the skin have been described in Part I of the series (10). The experimental arrangement for measuring the skin temperature is shown in Figure 1, and the parts of the

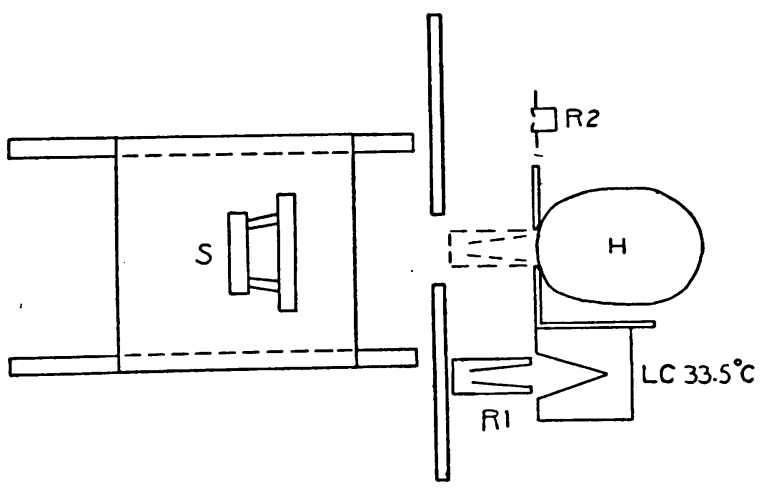

Fig. 1. Apparatus Used to Measure Stimulation and Heating of the Skin'by Radiation

$S=$ source of radiation, $H=$ head of subject, $R_{1}=$ radiometer for measuring skin surface temperatures, $L C$ $=$ Leslie Cube maintained at $33.5^{\circ} \mathrm{C} ., R_{2}=$ radiometer for measuring rate of radiation. 
apparatus used to measure the minimum stimulus are described in the first paper of this series. The radiometer, $R_{1}$, was mounted on a pendulum-like arm so that it could be swung rapidly from its resting position facing the Leslie Cube, to a position facing the skin surface. The construction of this radiometer and its use in measuring skin temperature have been previously described (5). In the present experiments the cone of a Leslie Cube served as a constant temperature reference body, and its temperature was maintained at about $33.5^{\circ} \mathrm{C}$. by a thermostatically controlled heater. Before each skin temperature measurement the temperature of the cube was read, and the difference between the cube and skin temperature measured directly from a galvanometer scale, calibrated so that a difference of $1^{\circ} \mathrm{C}$. caused a deflection of $56 \mathrm{~mm}$.

Skin temperature elevations produced by minimum stimulating amounts of radiation were so small that they were extremely difficult to measure, and such measurements were complicated by the fact that under ordinary environmental conditions the temperature of the skin is always slightly changing. To minimize this difficulty a thermal equilibrium was established between the skin and the air, walls, and other objects in the room before each experiment. When a satisfactory equilibrium had been established, measurements of skin temperature changes could be made with the assurance that whatever changes occurred were due to the experimentally applied radiation. A difference of $4^{\circ} \mathrm{C}$. in room temperature on different days did not appreciably effect the rise of skin temperature caused by radiation. Measurements were made on the forehead because it had been previously found to maintain a more constant temperature than any other part of the body. One other difficulty which interfered with the measurement of skin temperature was encountered, namely, visible and near infra-red radiation were reflected from the skin into the radiometer so that skin temperature could not be measured during irradiation.

To measure the rise of skin temperature die to radiation, the subject sat quietly in a room free from draughts for 10 to 30 minutes before the experiment began and during this interval skin temperature measurements were frequently made. After several successive readings gave the same temperature, the radiometer was returned to the Leslie Cube and the skin surface irradiated for the desired time. Immediately at the end of irradiation, the radiometer was moved back to the skin surface and measurements of skin temperature were made at 5 second intervals, for 60 seconds. From these data the curve for the cooling of the skin after irradiation was plotted. Four curves in Figure 2 show the cooling of the skin after it was irradiated for periods of $15,30,45$ and 60 seconds. By extrapolating these cooling curves back 8 seconds the skin temperature elevation due to the radiation can be read from the ordinate.

With this method, the results obtained on two subjects for the heating due to radiation were similar and could be repeated consistently. A large number of meas-

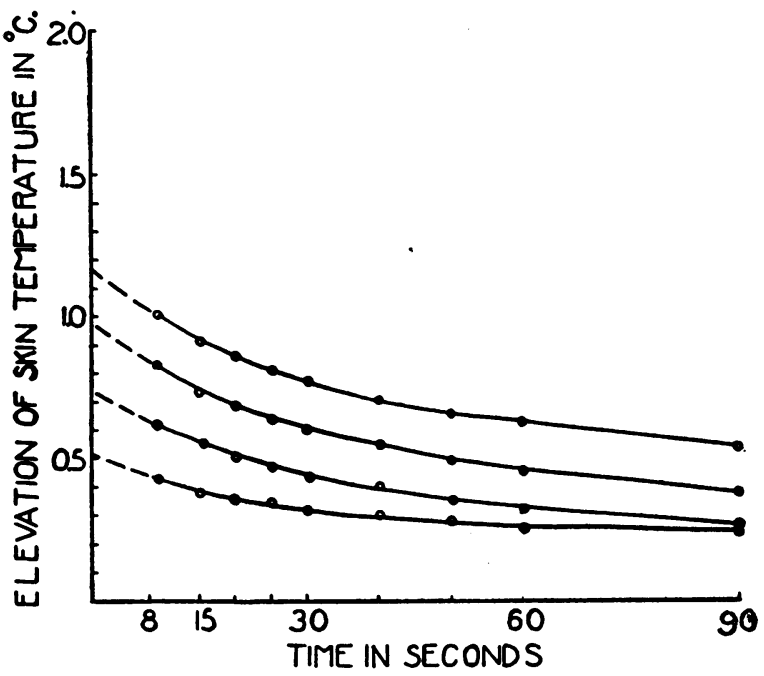

Fig. 2. Cooling of Skin Surface after Radiation 15 Seconds (Lower Curve); 20 Seconds (2Nd Curve); 45 Seconds (3rd Curve); 60 Seconds (Upper Curve).

urements with radiation of various intensities were made on these two subjects and the data have been considered together. The values have been recorded in Figure 3 for visible radiation, penetrating infra-red radiation, and nonpenetrating infra-red radiation. On this chart the intensity of radiation has been corrected for reflection and is plotted as the abscissa. The skin temperature elevation is recorded as the ordinate. Lines have been drawn between all the points plotted, and these lines indicate the average relationship between the strength of the radiation and the skin temperature elevation produced. The straight lines thus formed show that the temperature elevations are directly proportional to the radiant energy absorbed and demonstrate that the temperature elevations measured are due to the radiation and not to vasomotor or environmental influences.

We have called these charts "constant time charts" and have used them to plot the curves of the heating of the skin produced by any intensity of radiation in the experimental range. Such heating curves are shown in Figures 4, 5 and 6 in which a comparison has been made of the heating of the skin surface by visible, penetrating infra-red, and non-penetrating infra-red radiation.

\section{RESULTS}

In considering the heating of the skin by radiation it is necessary to distinguish between the amount of radiation received by the skin and the amount absorbed by the skin, a difference due to the reflection of visible and penetrating infra-red rays. White skin reflects about 35 per cent of the visible, 20 per cent of the penetrating infra-red, and practically none of the non-penetrating infrared radiation. Figure 4 shows the heating at the 


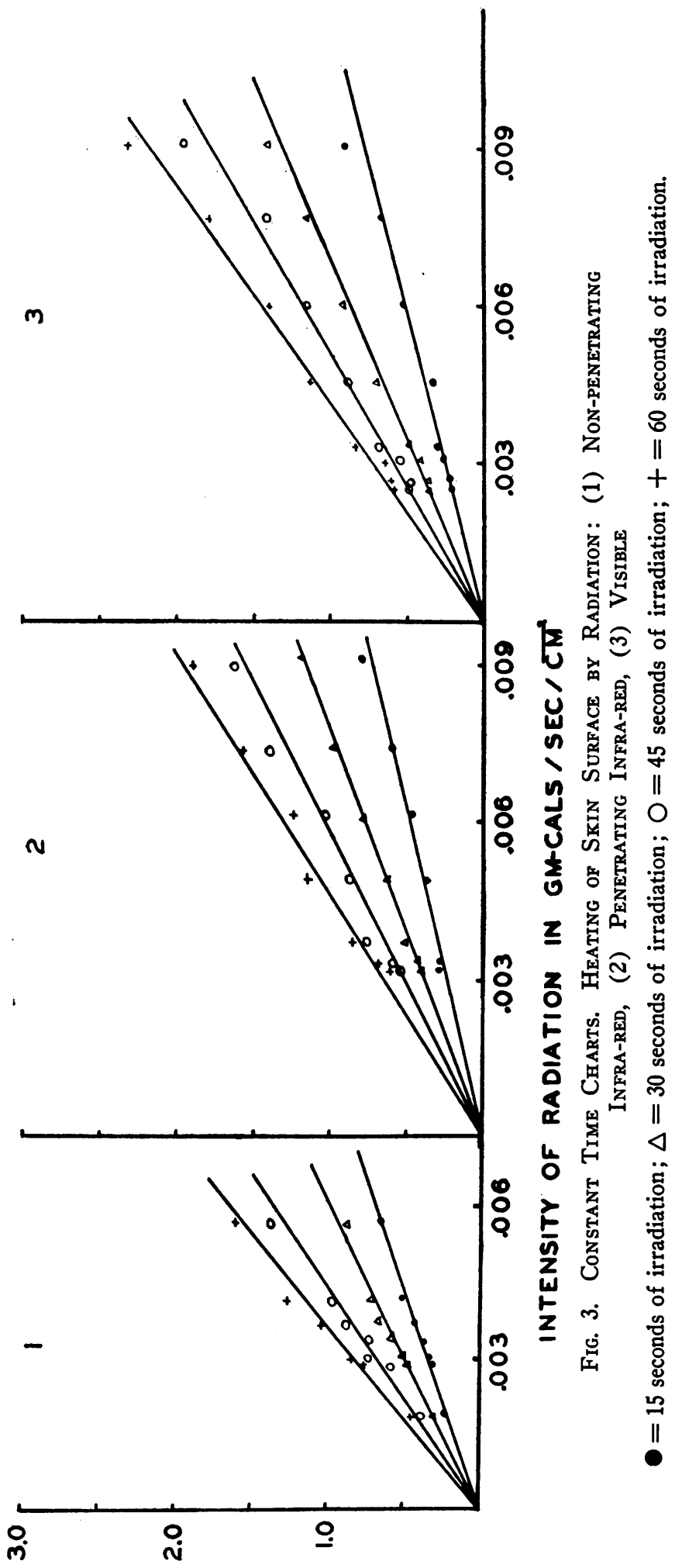

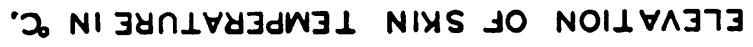




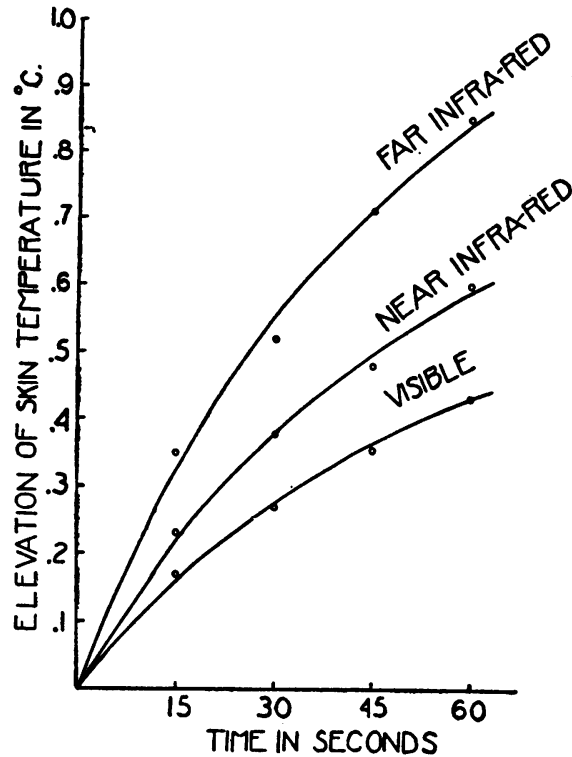

Fig. 4. Skin Surface Temperature Elevation Produced by the Same Incident Rate of Non-penetrating (Far) Infra-red, Penetrating (Near) Infra-red, and Visible Radiation

surface when the skin receives the same number of $\mathrm{gm} . \mathrm{cal} / \mathrm{cm}^{2} / \mathrm{sec}$. of radiation of the three types. The temperature is raised least by the visible radiation and most by the non-penetrating infra-red radiation. When the skin absorbs the same number of $\mathrm{gm}$. $\mathrm{cal} / \mathrm{cm}^{2} / \mathrm{sec}$. of these radiations the difference between the heating curves is, as shown in Figure 5, less marked.

From the constant time charts we determined the number of $\mathrm{gm} . \mathrm{cal} / \mathrm{cm}^{2} / \mathrm{sec}$. of the three types of radiation which must be absorbed to produce the same curve for the heating of the skin. The rates of radiation were: visible .0035 , penetrating infra-red .0031, and non-penetrating infra-red .0026 . When the sources were adjusted so that the skin of the subjects absorbed these amounts of radiation there was a striking difference in the sensation evoked. The non-penetrating infra-red felt much warmer than the penetrating infra-red, and the penetrating infra-red produced a definitely warmer sensation than the visible radiation.

The minimum rates of radiation necessary to produce sensation were determined on the same two white subjects on whom the heating measurements for the constant time charts were made. An area of $7.5 \mathrm{sq} . \mathrm{cm}$. on the forehead was exposed to the radiation. The results of a series of

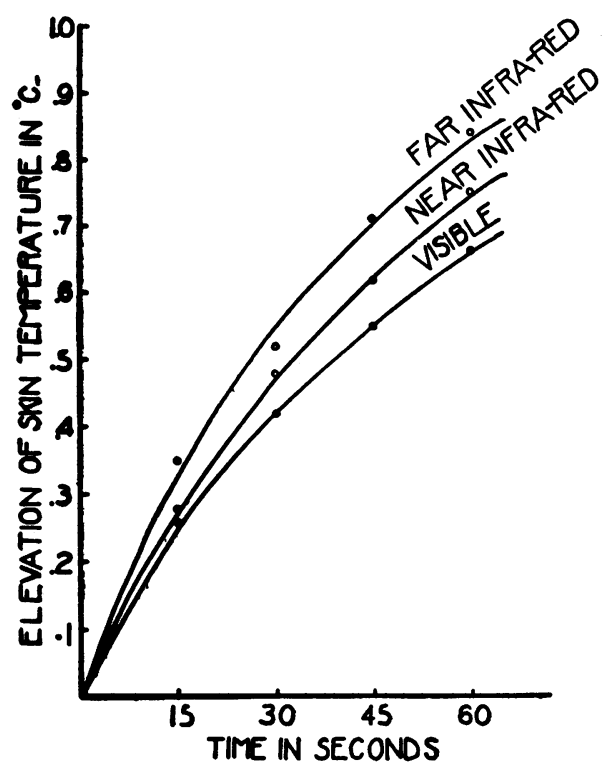

Fig. 5. Skin Surface Temperature Elevations Produced by the Absorption of the Same Rate of Non-penetrating (Far) Infra-red, Penetrating (NeAr) INFra-Red, and Visible Radiation

tests are shown in Table I. The average values for the two subjects are: visible radiation .0035 $\mathrm{gm}$. cal $/ \mathrm{cm}^{2} / \mathrm{sec}$., penetrating infra-red $.0025 \mathrm{gm}$. $\mathrm{cal} / \mathrm{cm}^{2} / \mathrm{sec}$., and non-penetrating infra-red .0017

TABLE I

Minimum stimuli in $\mathrm{gm}$. $\mathrm{cal} / \mathrm{cm}^{2} / \mathrm{sec}$. of radiation-corrected for reflection

\begin{tabular}{c|c|c|c|c|c|c}
\hline \hline & \multicolumn{2}{|c|}{ Visible } & \multicolumn{2}{c|}{ Near infra-red } & \multicolumn{2}{c}{ Far infra-red } \\
\hline Subject......... & I & II & I & II & I & II \\
\hline & .0032 & .0034 & .0023 & .0027 & .0017 & .0019 \\
.0035 & .0036 & .0023 & .0026 & .0016 & .0016 \\
& .0036 & .0035 & .0026 & .0025 & .0020 & .0016 \\
& .0035 & .0035 & .0026 & .0024 & .0014 & .0016 \\
\hline Average ........ & .0037 & .0035 & .0026 & .0024 & .0017 & .0016 \\
\hline
\end{tabular}

gm. cal $/ \mathrm{cm}^{2} / \mathrm{sec}$. of radiation. The heating produced by these amounts af radiation is shown in Figure 6 . The temperature is raised most by the visible radiation and least by the non-penetrating infra-red. In the following section an hypothesis is suggested to account for the results.

\section{COMMENT}

There is a difference between the surface and deep temperature changes produced by various 
types of radiation because rays of certain wavelength are reflected more or allowed to penetrate deeper than others. Correction for energy loss by reflection shows that there is an important difference in surface heating dependent on the degree of penetration. When all of the energy is

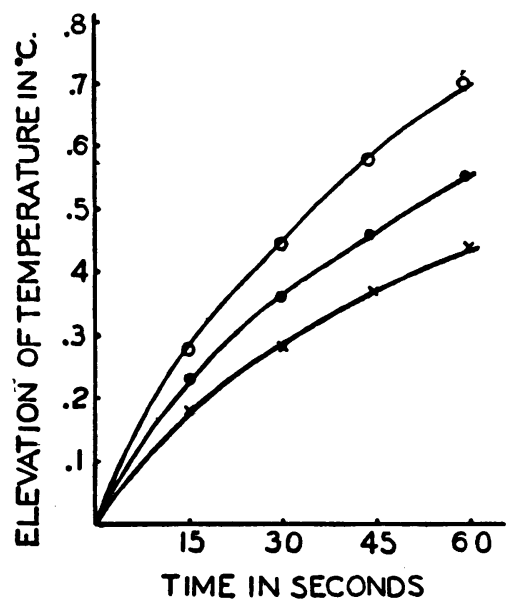

Fig. 6. Skin Surface Temperature Elevations Produced by the Minimum Stimulating Rates of Visible (Upper Curve), Penetrating Infra-Red (Middle Curve), and Non-Penetrating Infra-Red RadiaTION (LOWER CURve)

absorbed at the skin surface a high surface temperature elevation occurs, and the greater the penetration, the less the surface temperature is elevated. In the deep layers of the skin the temperature is raised higher by the penetrating than by the non-penetrating radiations, a fact illustrated by the experiments of Sonne (11), Keller (8), and Loewy and Dorno (9) who measured the surface and deep temperature during visible, penetrating infra-red, and non-penetrating infra-red radiation. Knowing the penetrability of a radiation it is thus possible to estimate the deep temperature elevation from the surface temperature rise, assuming that there is little or no change in conduction of heat during a short interval of time.

The skin of white subjects reflects 35 per cent of visible radiation and over half of the incident energy penetrates more than $0.2 \mathrm{~mm}$. into the skin. Twenty per cent of the penetrating infra-red radiation is reflected, and less than half of the incident energy penetrates beyond $0.2 \mathrm{~mm}$. Nonpenetrating infra-red is not reflected and is completely absorbed at the skin surface. The thermal
EQUAL RADIATION

EQUAL SENSATION
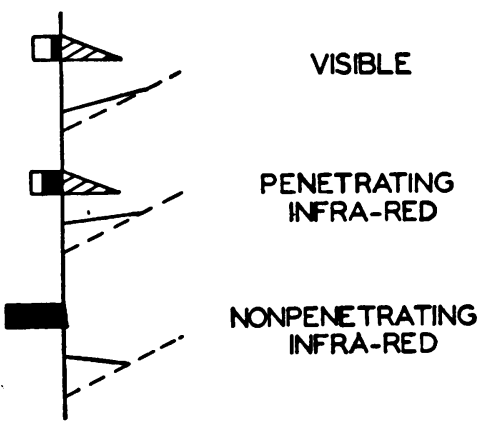

SURFACE DEEP

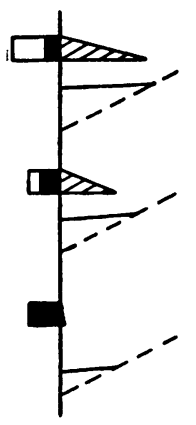

SURFACE DEEP

Fig. 7. Relative Temperature Changes Produced in the Skin by Radiation

Interrupted lines indicate normal thermal gradient; solid lines, changes produced by the absorption of radiation. Horizontal columns indicate calories of radiant energy. White area is reflected calories; solid area, calories absorbed at skin surface; ruled area, calories absorbed below skin surface.

changes produced in the skin by visible, penetrating infra-red, and non-penetrating infra-red radiation are shown diagrammatically in Figure 7. The strength of the radiant energy is represented by horizontal columns in which the energy reflected is the white part, that absorbed at the skin surface is the solid part, and that which penetrates into the skin is the ruled part. The normal temperature gradient in the skin is indicated by the interrupted lines, and the estimated changes due to the absorption of radiation are shown by the solid lines.

On the left side of this diagram the changes produced by the same incident strength of these three types of radiation are shown. With visible radiation, a small surface temperature elevation is associated with a relatively large deep temperature rise; with penetrating infra-red radiation there is a larger surface temperature elevation and a relatively smaller deep temperature rise; with nonpenetrating infra-red radiation the surface temperature elevation is greatest and the deep temperature elevation is relatively smallest.

The right side of this diagram shows the thermal changes produced in the skin by the minimum strength of these three types of radiation which is capable of producing a sensation of warmth. A large amount of visible radiation is needed to 
stimulate sensation and high surface and deep temperature elevations are produced. With the penetrating infra-red stimulus the surface and deep temperature elevations are smaller than with the visible stimulus. The non-penetrating infrared stimulus produces the smallest deep and surface temperature elevations.

The temperature changes 'produced in the skin by these types of radiation have been considered in detail because a thorough understanding of them is necessary to determine what thermal change is responsible for the stimulation of the heat end organs. Warm sensation depends on one of three possible thermal changes in the skin: the degree of temperature rise, the rate of temperature rise, or a change (or rate of change) of the normal temperature gradient in the skin. The sensation resulting from radiation is due to the warming produced by the absorption of the energy. The same sensation produced by these three types of radiation should therefore be associated with a common thermal change in the skin. It is evident from Figure 7 that minimal sensation resulting from these three types of radiation is associated with a markedly different degree of surface and deep temperature rise. It is also associated with a different rate of temperature rise. The temperature changes shown on Figure 7 are those which occur when the sensation is recognized, that is, after three seconds of radiation. Since the duration of radiation was the same, a difference of actual temperature rise must be accompanied by a difference of rate of temperature rise. Thus, neither the degree of temperature change in the skin nor the rate of temperature change is constant when the same sensation is evoked by these three types of radiation, and neither of these thermal changes can be that which activates the heat end organs.

The only other thermal alteration which could activate the end organs is a change of the normal temperature gradient in the skin. A constant decrease of the temperature gradient in the skin would be shown in Figure 7 by a parallel direction of the solid lines. These three types of radiation are absorbed by the skin in such a way that we would expect them to cause such a constant decrease of the normal thermal gradient with minimal sensation. Our results thus support the hy- pothesis that a decrease of the normal temperature gradient in the skin is responsible for the sensation of warmth.

This interpretation is open to the criticism that no actual measurements were made of the temperature below the skin surface. We do not believe that it is technically possible to measure the small changes of the thermal gradient which accompany the minimal sensation of warmth. The histological structure of the skin with its vascular and avascular layers does not permit the assumption that the normal thermal gradient is uniformly distributed within the depth of the tissue. Therefore, no calculation of the temperature gradient between two widely separated thermocouples in the tissue is warranted. Local temperature differences can be measured by thermocouples but the only temperature receptors which have been identified lie at the depths of $0.1 \mathrm{~mm}$. and $0.3 \mathrm{~mm}$., and it is not possible to place small wires accurately at these levels. Furthermore, the presence of thermocouples in the skin interferes with the normal temperature relationship. For these reasons we feel that a study of the gradient hypothesis must be indirectly approached and that our experiments have been performed under more natural conditions than those previously reported.

The application to our results of known facts about the heating below the skin surface by these three types of radiation has lead us to support the hypothesis that the stimulation of warm sensation depends on a change in the normal temperature difference between deep and superficial temperature receptors in the skin.

\section{SUM MARY AND CONCLUSIONS}

The heating of the surface of the skin of white subjects by visible radiation, penetrating infra-red radiation, and non-penetrating infra-red radiation has been measured by a radiometer. It was found that:

1. With the same incident strength of these three types of radiation the skin surface temperature is elevated highest by the non-penetrating infra-red, and least by the visible radiation. Part of this difference is due to the reflection of visible and penetrating infra-red rays from the skin. After correction for reflected radiation there is a smaller difference between the heating of the skin 
surface by visible, penetrating infra-red, and nonpenetrating infra-red radiation.

2. When minimum perceptible sensation is produced by these three types of radiation the skin surface temperature is elevated higher by the visible stimulus than by the penetrating infra-red stimulus, and higher by the penetrating infra-red stimulus than by the non-penetrating infra-red stimulus.

The application to our results of known facts about the heating below the skin surface by these three types of radiation has led us to conclude that the stimulation of warm sensation depends on a decrease of the normal thermal gradient in the skin and not on the degree or rate of temperature change in the skin.

\section{BIBLIOGRAPHY}

1. Bazett, H. C., and McGlone, B., Temperature gradients in the tissues in man. Am. J. Physiol., 1927, $82,415$.

2. Bazett, H. C., McGlone, B., Williams, R. G., and Lufkin, H. M., Sensation. I. Depth, distribution and probable identification in the prepuce of sensory end-organs concerned in sensations of temperature and touch; thermometric conductivity. Arch. Neurol. and Psychiat., 1932, 27, 489.

3. Bazett, H. C., and McGlone, B., Studies in sensation. II. The mode of stimulation of cutaneous sensations of cold and warmth. Arch. Neurol. and Psychiat., 1932, 27, 1031.
4. Ebbecke, U., Uber die Temperaturempfindungen in ihrer Abhängigkeit von der Hautdurchblutung und von den Reflexzentren. Arch. f. d. ges. Physiol., 1917, 169, 395.

5. Hardy, J. D., The radiation of heat from the human body. I. An instrument for measuring the radiation and surface temperature of the skin. J. Clin. Invest., 1934, 13, 593.

6. Hardy, J. D., The radiation of heat from the human body. II. A comparison of some methods of measurements. J. Clin. Invest., 1934, 13, 605.

7. Hering, M. E., Grundzüge einer Theorie des Temperatursinns. Setzungsb. der Kaiser. Akad. d. Wissensch., Wein, 1877, 75, 101.

8. Keller, P., Die Strahlen-Wärmereaktion der Haut. II. Die Temperaturverhältnisse in der wärmebestrahlten Haut. Strahlentherapie, 1929, 33, 711.

9. Loewy, A., and Dorno, C., Uber Haut- und Körpertemperaturen und ihre Beeinflussung durch physikalische Reize. Strahlentherapie, 1925, 20, 411.

10. Hardy, J. D., and Oppel, T. W., Studies in temperature sensation. I. A comparison of the sensation produced by infra-red and visible radiation. $J$. Clin. Invest., 1937, 16, 517.

11. Sonne, C., The mode of action of the universal light bath. V. Visible and invisible heat rays, calculations and determinations of the temperature of the subcutaneous tissue during radiation with luminous rays and obscure heat rays of the maximum intensity endurable. Acta. med. Scandinav., 1921, 54, 374.

12. Weber, E. H., and Wagner, R., Braunschweig, F. Vieweg und Sohn. Handwörterbuch der Physiologie, 1846, 3, 549. 